\section{La influencia}

\section{del Banco}

Mundial

\section{en las politicas educativas de Colombia}

\section{A influência do Banco Mundial nas politicas educacionais da Colômbia}

\section{The Influence of the World Bank on the Educational Policies in Colombia}

\section{Adolfo León Atehortúa Cruz}

* Profesor titular del Departamento de Ciencias Sociales y decano de la Facultad de Humanidades de la Universidad Pedagógica Nacional, Bogotá, Colombia. Licenciado en Historia por la Universidad del Valle, magister en Historia por la Universidad Nacional de Colombia y doctor en Sociología de la Escuela de Altos Estudios en Ciencias Sociales de París.

Correo electrónico. adolfoatehortua@cablenet.net.co
El artículo procede de una revisión de las políticas del Banco Mundial, en contraste con las políticas educativas agenciadas en Colombia, especialmente en el contexto de la educación superior. La reflexión fue presentada en escenarios universitarios, a propósito del debate académico a la reforma de la educación superior propuesta por el gobierno de Santos, que fue retirada posteriormente por efectos de la movilización estudiantil, en 2011.

\section{Resumen}

El artículo ofrece una visión de síntesis en torno a las políticas educativas diseñadas por el Banco Mundial y su reciente aplicación en Colombia. Ubica en ese enfoque a la llamada Revolución Educativa y enfatiza en las últimas decisiones gubernamentales con respecto a la educación superior. Se destaca, asimismo, la concepción que sobre educación reposa en los Planes Nacionales de Desarrollo y las estrategias previstas para el impulso de las políticas enunciadas que agencian un enfoque mercantilizador, el cual incide en las tendencias privatizadoras de la educación pública.

\section{Palabras clave}

Políticas educativas, Banco Mundial, educación, competencias, Revolución Educativa.

\section{Resumo}

0 artigo oferece uma visão de síntese acerca das políticas educacionais construídas pelo Banco Mundial e sua recente aplicação na Colômbia. Localiza tal enfoque na chamada Revolução Educativa, e destaca as últimas decisões governamentais referidas à educação superior. Salienta-se, do mesmo modo, a concepção sobre educação que aparece nos Planos Nacionais de Desenvolvimento e nas estratégias previstas para impulsionar as políticas enunciadas que mobilizam um enfoque mercantilizador, o qual incide nas tendências privatizadoras da educação pública.

\section{Palavras chave}

Políticas educacionais, Banco Mundial, educação, competências, Revolução Educativa.

\section{Abstract}

This article offers a summary vision around the educational policies designed by the World Bank and their recent application in Colombia. It locates in that approach they call Educational Revolution and emphasizes in the last governmental decisions related to higher education. It is underlined, likewise, the conception about education that it is reposed in the National Plans of Development, and the previewed strategies to promote the named policies, which get a mercantilist focus that has a serious incidence in the privatizing tendencies of public education.

\section{Key words}

Educational policies, The World Bank, education, competencies, Educational Revolution.

Fecha de recepción: 12 de marzo de 2012 Fecha de aprobación: 30 de mayo de 2012

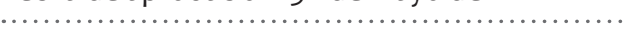

Pedagogía y Saberes No. 36 Universidad Pedagógica Nacional Facultad de Educación. 2012, pp. 69 - 79 


\section{E1 Banco Mundial y la educación en Colombia}

$\mathrm{E}$ n 1966, el Banco Mundial hizo por primera vez en su historia un préstamo dirigido al sector educativo en Chile. Treinta años más tarde, no solo se había convertido en el mayor prestamista para las inversiones educativas en los países de América Latina y el Caribe sino, también, en el gran interventor para el diseño de políticas públicas en ese ámbito. En criterio del Banco, por esa razón, era necesario revisar a fondo los designios crediticios, inmiscuirse en la construcción de orientaciones y estrategias dirigidas al sector y comprometer a los líderes políticos en una reforma educativa profunda.

En efecto, en abril de 1998, la II Cumbre de las Américas reunió en Santiago de Chile a presidentes de los países del hemisferio occidental y obtuvo de ellos su responsabilidad para "una agenda educativa común y ambiciosa". Como resultado inmediato de la Cumbre, el compromiso se confirmó con una reunión de ministros de Educación y líderes del sector privado de América Latina y el Caribe, convocada por el presidente del Banco Mundial y celebrada en junio de 1998 en la sede central de la entidad en Washington. El camino para elaborar una nueva política educativa en toda la región quedó dispuesto.

Bajo dicha perspectiva, el Banco realizó consultas y talleres con expertos designados por los ministerios de Educación del área, en donde "salieron a relucir consejos importantes sobre las prioridades del Banco dentro del sector de educación" y acerca de los procesos utilizados para el desarrollo de proyectos y la ejecución de estudios. A partir de allí, se elaboró una estrategia educativa para América Latina y el Caribe, aprobada por la junta directiva del Banco en abril de 1999.

De acuerdo con los postulados del Banco Mundial, se trataba de "combinar el conocimiento y la experiencia global con la capacidad para ayudar financieramente a América Latina y el Caribe en la confrontación de los desafíos educativos de la próxima década". Como objetivo general, el Banco se proponía "encontrar las soluciones a los problemas difíciles para reducir la pobreza a través del crecimiento económico y la asignación de inversiones y servicios focalizados en los más pobres". La meta, en conclusión, consistía en "levantar, sobre todo, el capital humano de la región".

Las estrategias que señaló el Banco en este primer documento, "Educational change in Latin America and the Caribbean" (World Bank, 1999), fueron las siguientes:

\section{a. Incluir a los excluidos}

El Banco dirigiría su principal esfuerzo hacia inversiones en los programas de niñez temprana, alimentación y salud escolar. Buscaría optimizar la asistencia escolar a través de incentivos financieros entregados a familias pobres, e incrementar las oportunidades crecientes para educación secundaria y terciaria a través de becas ajustadas a ingresos.

\section{b. Elevar la calidad pedagógica}

El Banco promovería mejoras radicales en el entrenamiento del maestro, introduciría su evaluación periódica y proporcionaría herramientas eficaces de evaluación estudiantil para diagnosticar su rendimiento.

\section{c. Mejorar la transición de la escuela al mundo adulto}

El Banco propondría la transición de la escuela al mundo adulto ajustando el volumen de la educación secundaria y equipando a la juventud con una formación adecuada para la participación en el trabajo y en la sociedad.

\section{d. Optimización y descentralización de recursos}

El Banco impulsaría la reingeniería de los ministerios de Educación y apoyaría reformas en la gestión y mejoras de información para asegurar la rendición de cuentas. Así mismo, asistiría a los países en la identificación de los cambios en los incentivos que podrían modificar el comportamiento de los proveedores y afectar la continuidad de las iniciativas de reforma.
Uribe Vélez, la contratación de la prestación del "servicio educativo" se convirtió en la fórmula expedita para abandonar paulatinamente las obligaciones del Estado y trasladar a las instituciones y a los particulares, la obligación de autofinanciar la educación.

\section{e. Educación terciaria}

El Banco induciría reformas a la educación superior para garantizar su calidad y eficacia, mejorar el acceso con ampliación de cobertura y fortalecer el papel 
integral del sector privado en el financiamiento y la prestación del servicio educativo.

\section{f. Estimular y evaluar las innovaciones educacionales}

Finalmente, el Banco estimularía y evaluaría las innovaciones educativas, sobre todo en el uso de tecnologías, para identificar maneras más rentables de utilizar los avances en las TIC, aumentar el acceso y mejorar la calidad.

Con estas condiciones, Colombia suscribió los primeros compromisos impulsados desde la alcaldía de Enrique Peñalosa en Bogotá (1998-2000), bajo la dirección de su secretaria de Educación, Cecilia María Vélez White. La funcionaria no solo participó en los acuerdos con el Banco Mundial sino que hizo gala de su experiencia como subdirectora de Planeación Nacional y directora de la Unidad de Desarrollo Territorial en el Departamento Nacional de Planeación. En el escenario nacional, Francisco José Lloreda, ministro de Educación de Andrés Pastrana (1998-2002) y exdirector de Planeación Nacional, hizo lo propio.

En cumplimiento de las directrices del Banco, surgieron así las propuestas de "reorganización institucional y optimización de la capacidad instalada" y se lanzaron el "Plan Estratégico" del Ministerio de Educación y el "Plan de reorganización del sector educativo" (PRESE), los cuales fueron coadyuvados por el Ministerio de Hacienda y el Departamento Nacional de Planeación en el año 2000.

Bajo el pretexto de la racionalización y el aprovechamiento máximo de los recursos, se inició la ampliación de la relación maestro-alumno, al precio de un verdadero hacinamiento en las aulas escolares; se extendieron los convenios de desempeño con los departamentos bajo el principio del subsidio a la demanda, se incrementó la cobertura, se fusionaron y cerraron instituciones, se suprimieron las direcciones de escuelas y se eliminó la repetición de grados. La mayoría de las prioridades trazadas por el Banco fueron atendidas.

No obstante, en la práctica crediticia, el Banco Mundial extremó sus posturas, puso de relieve las tendencias y desafíos que, en su opinión, motivaban la reevaluación del sector de educación en América Latina y, desde luego, condicionaban sus préstamos:

Los rápidos cambios económicos y tecnológicos están exigiendo que los gobiernos de la región inviertan en las habilidades y la educación de la fuerza laboral.

El mayor acceso a la educación es la clave para reducir la persistente pobreza y desigualdad social en los países de la región.

Para que las nuevas democracias de la región prosperen, deben contar con la amplia participación de una ciudadanía educada e informada.

Los cambios en el papel y la organización del Estado significan que el gobierno central ya no es el único responsable de prestar servicios educativos, sino que cada vez más comparte esa función con los gobiernos locales, las comunidades, las familias, las personas individuales y el sector privado. (World Bank, 1999, p. 27)

Nacieron de allí el Acto Legislativo $01 \mathrm{y}$ la Ley 715 o de transferencias, con los cuales se reformaron los artículos 356 y 367 de la Constitución Política Nacional, se destruyó el situado fiscal ya congelado y reducido desde el gobierno de Ernesto Samper, y se impuso un nuevo esquema para la asignación de recursos en la educación pública: la municipalización y plantelización de la financiación educativa, el subsidio a la demanda.

El Decreto 1528 del 24 de julio de 2002, por el cual se reglamentaron parcialmente los artículos 23 y 27 de la Ley 715 y se viabilizó la contratación con entidades privadas, entregando incluso establecimientos del Estado, constituyó, igualmente, un componente más que acataba las recomendaciones del Banco. Se sumó a ello un nuevo estatuto docente y un nuevo sistema de inspección y vigilancia impuestos al final de la administración Pastrana. Este último, plasmado en el Decreto 1283 de 2002, privilegió a la empresa privada educativa pero persiguió, desde su esquema tecnocrático de evaluación de desempeño y competencias, a las instituciones y docentes públicos.

Acto seguido, las políticas y estrategias se apuntalaron aún más con la confirmación de Cecilia María Vélez como secretaria de Educación de la administración de Antanas Mockus (2001-2003) y con su 
presencia de ocho años en el Ministerio de Educación bajo el gobierno de Álvaro Uribe Vélez (2002-2010).

\section{La Revolución Educativa}

Muy temprano, en las "Bases del Plan Nacional de Desarrollo 2002-2006", la educación se definió como "factor esencial del desarrollo humano, social y económico y un instrumento fundamental para la construcción de equidad social" (DNP, 2007, p.122). Desaparecieron muchas ofertas que el candidato Uribe Vélez había inscrito en su programa. La capacitación y la investigación científica, por ejemplo, se borraron como estrategias frente al desafío. Al lado de la cobertura, la calidad y la pertinencia, apareció una nueva que era, en realidad, la más antigua de todas: "la eficiencia", un programa que correspondía a los antiguos planes de la administración Pastrana.

La calidad tampoco se supuso edificada sobre la capacitación de los docentes. Se volvió a los estándares, a las evaluaciones homogéneas y sancionatorias. La conectividad no se citó como componente claro de la Revolución Educativa y registró un aislado lugar en ciencia y tecnología. La pertinencia laboral se esgrimió menos como estímulo y más como instrumento para el recorte presupuestal de las universidades. El presupuesto de investigación, prometido en el $1 \%$ del PIB, quedó en la meta del $0.6 \%$ para el final del cuatrenio, incluyendo la inversión privada.

Las "Bases del Plan Nacional de Desarrollo", consideradas borrador inicial del Plan, partieron de un diagnóstico crítico en tres ámbitos: cobertura, calidad y eficiencia. Según el documento, a pesar de los avances obtenidos durante los últimos tiempos, la estadística nacional presentaba un porcentaje considerable de la población en edad escolar por fuera del sistema educativo y una marcada inequidad en las posibilidades de acceso y permanencia. La calidad era deficiente y su deterioro se observaba creciente en los grados de formación básica y media. Además, el diseño institucional educativo no generaba incentivos a la eficiencia y limitaba el avance en la expansión de cobertura y mejoramiento de la calidad (Cfr. Atehortúa, 2006).

Con base en tal diagnóstico, la propuesta formuló cinco programas detallados a continuación:

1. Ampliar la cobertura en educación preescolar, básica y media, a través de la reorganización ins- titucional y mediante la definición de estándares técnicos que tuvieran en cuenta el número de niños atendidos.

2. Ampliar la cobertura en educación superior, aumentando la eficiencia en el uso de los recursos físicos, humanos y financieros de las instituciones públicas. Además de la integración de instituciones educativas mediante convenios y fusiones, sobresalía entre los mecanismos fundamentales la concertación de planes de desempeño que definieran la relación de personal administrativo por docente y estudiante, y determinaran el tamaño óptimo de la planta de cargos, así como la asignación académica de los docentes, ajustada a estándares definidos también por el gobierno y las instituciones. Tras modificar el sistema de asignación de recursos establecido por la Ley 30 de 1992, un nuevo fondo unificaría todos los recursos y los asignaría de acuerdo con las metas y logros alcanzados en los planes de desempeño pactados con el gobierno por cada institución educativa.

3. Mejorar la calidad de la educación preescolar, básica y media, con mecanismos ligados a estándares de evaluación y universalización, acompañamiento de las instituciones que presenten bajos resultados, pertinencia educativa y diseño de estrategias para el uso y racionalización de los materiales educativos.

4. Mejorar la calidad de la educación superior mediante la pertinencia de los programas impartidos, creación del observatorio laboral, acreditación de los programas e instituciones, establecimiento de estándares mínimos para todos los programas y aplicación de los exámenes de calidad ECAES a todas las carreras, de manera obligatoria.

5. Mejorar la eficiencia del sector educativo impulsando la modernización de la administración y de la gestión en el sector educativo.

\section{La educación superior}

Bajo el gobierno de Uribe Vélez, la contratación de la prestación del "servicio educativo" se convirtió en la fórmula expedita para abandonar paulatinamente las obligaciones del Estado y trasladar a las instituciones y a los particulares, la obligación de autofinanciar la educación. La autonomía escolar se propuso como 
"autonomía real" de carácter financiero y de manejo de personal; huelga decir, autofinanciación.

Con respecto a la educación superior y en concordancia con los programas del Banco Mundial, la nueva política planteó la "cobertura universal, la buena calidad de la educación y el acceso democrático". Sin embargo, en sus contenidos concretos, un aspecto definió el desafío más importante: la pertinencia laboral. En este punto incluyó la vinculación de la universidad a la actividad productiva y prometió exigirle información pública certificada sobre el éxito laboral o empresarial de sus egresados, y una integración de los programas de pregrado a las incubadoras de las empresas para estimular la creatividad empresarial de los estudiantes (Cfr. Uribe, 2001).

Poco a poco, los créditos contratados con el Banco Mundial para el sector educativo definieron las orientaciones públicas. Colombia obtuvo un cronograma de desembolsos pero obtuvo también claros compromisos en política educativa (World Bank, 2002).

Conforme a ulteriores escritos académicos, las políticas y estrategias del Banco Mundial se concretaron en sus aplicaciones y se entrelazaron con sugerencias similares planteadas desde el BID ${ }^{1}$. Para el año 2002, en las propuestas del Banco, sobresalían cuatro aspectos:

1. Financiamiento de una educación por competencias inspirada en la competitividad y el desempeño, para incrementar la cobertura en áreas de interés productivo y disciplinas con impacto directo sobre el mercado laboral.

2. Construcción de mecanismos para asegurar el ajuste del sistema, tanto en lo económico como en el control a través de la autoevaluación, la acreditación y la implementación de estándares "indicadores de calidad".

3. Creación de observatorios del mercado laboral ofrecido por las instituciones de educación públicas y privadas, bajo patrones de eficiencia.

4. Créditos educacionales orientados a subsidiar la demanda y a fortalecer la injerencia privada en la educación ${ }^{2}$.

Al lado de dichos aspectos, el Banco Mundial concretó dos objetivos para la educación superior: uno, crear un mercado para dicho nivel educativo,

1 Cfr. Inter-American Development Bank (2000). Reforming Primary and Secondary Education in Latin America and the Caribbean. An IDB Strategy. Washington, D.C.: Sustainable Development Department Sector Strategy and Policy Papers Series. [en línea] http://idbdocs.iadb.org/wsdocs/ getdocument.aspx?docnum

2 Los escritos que contienen los aspectos enunciados están disponibles en línea: http://scholar.google.com.co/scholar? $\mathrm{q}=$ Educational+change+in+Latin+America y dos, mejorar la capacidad del sector público para atenderlo.

Las estrategias que el mismo Banco propuso aplicar pasaron por el incremento de la capacidad institucional y de políticas, el financiamiento de fórmulas que permitieran aumentar la cobertura y afirmar disciplinas de impacto social que no fueran asumidas por las instituciones privadas, el aseguramiento de la calidad a través de evaluaciones y aplicación de estándares, los observatorios del mercado laboral para orientar la demanda hacia las oportunidades, y la oferta de créditos educacionales con subsidios focalizados en los sectores de población más pobre.

Finalmente, advirtió el Banco: "Sin una reforma económica generalizada que tenga como objetivo promover la estabilidad macroeconómica, el libre comercio, un entorno propicio para las inversiones y mercados laborales efectivos, el valor económico de la educación terciaria pierde gran parte de su potencial" (Lauritz Holm-Nielsen et.al., 2003, p. 3).

Colombia fue fiel a los designios. Las decisiones gubernamentales se plasmaron sucesivamente en los Planes Nacionales de Desarrollo, a partir de la misma concepción de educación.

\section{La concepción de educación en los Planes Nacionales de Desarrollo}

En los Planes Nacionales de Desarrollo 2002-2006, 2006-2010 y 2010-2014, la piedra angular en materia educativa partió de la definición que sobre la educación y su papel adoptó cada uno de los planes.

Muy temprano, en el Plan 2002-2006, la educación se definió como "factor esencial del desarrollo humano, social y económico y un instrumento fundamental para la construcción de equidad social" (DNP, 2007, p. 122). He aquí las variables permanentes:

$$
\begin{gathered}
\text { Educación } \longrightarrow \text { Factor; } \\
\text { Educación } \longrightarrow \text { Instrumento; } \\
\text { Educación = Equidad }
\end{gathered}
$$

La visión, desde luego, es utilitarista y reemplaza la ecuación:

$$
\text { Educación }=\text { Derecho }
$$

Esta ecuación es precisamente establecida por nuestra Constitución Nacional en el Título II, artículos 44, 45 y 67: "La educación es un derecho fundamental de niños y jóvenes que debe ser asistido y garantizado por el Estado; un derecho de la persona y un servicio público con función social que se ofrecerá en forma obligatoria entre los cinco y quince años de edad, y gratuita en las instituciones del Estado". 
El Plan Nacional de Desarrollo 2006-2010 volvió sobre las variables utilitaristas (DNP, 2007 Tomo I, p. 150). Definió la educación como "un instrumento para lograr la inclusión social por características diferentes a las que tienen que ver con su nivel socioeconómico". Es decir:

\section{Educación $\longrightarrow$ Instrumento; \\ Educación = Inclusión social}

El Plan Nacional de Desarrollo propuesto por el presidente Santos insistió en que la educación "es el instrumento más poderoso para reducir la pobreza y el camino más efectivo para alcanzar la prosperidad" (DNP, 2011). Las variables serían:

\section{Educación $\longrightarrow$ Instrumento; \\ Educación = Reducción de pobreza;

$$
\text { Educación = Prosperidad }
$$

Las coincidencias saltan a la vista. Pero no solo en la concepción. La visión utilitarista de la educación trae consigo una visión estrecha en las estrategias.

En cada uno de los Planes el papel estratégico de la educación se ubicó en el incremento de las capacidades y destrezas personales, léase competencias, para el empleo y la productividad.

Educación $\longrightarrow$ Instrumento

Competencias $=$ Equidad $=$ Inclusión social $=$ Prosperidad

Así lo expresa claramente el Plan 20062010: la "adquisición de conocimientos, competencias y habilidades productivas" es requisito para "contribuir al crecimiento e incrementar la productividad del empleo" (DNP, 2007, p. 152).

Además del utilitarismo, subyace a tales definiciones y consideraciones una visión economicista que no solo aplaza la inclusión social y la equidad para el "futuro", sino que oculta las auténticas razones de la desigualdad y elude la responsabilidad del Estado frente a ellas.

Según la visión de estos Planes, las diferencias sociales no son producto de categorías históricas referidas a contenidos reales y empíricamente observables, presentes en la evidencia misma (Thompson, 1979, pp. 36-37), sino más bien accidentes producidos por la falta de educación y de destrezas para el empleo.
Se omite la existencia de diferencias crónicas en la infraestructura de las sociedades cuyo reconocimiento, como piensa Giddens, no significa estar de acuerdo con una primacía causal, universal y necesaria, de los propios factores infraestructurales: en muchas ocasiones, las influencias específicamente políticas condicionan a la vez que expresan dichas diferencias (Giddens, 1979, pp. 21-22).

Decía el entonces presidente Uribe que la educación en tanto "instrumento para mejorar el ingreso y aumentar la productividad", "genera movilidad social y evita la condena a ser pobre por herencia" (Uribe, 2001 y 2002). En otras palabras, formación en términos instrumentales de "éxito y eficiencia", "formación para triunfar": a mayor y mejores competencias, más riqueza, más éxito.

Podría responderse, con sencillez, que ese tipo de formación no es formación, es capacitación y se limita, por tanto, a las cualidades y destrezas que el individuo necesita para defenderse en el caos salvaje del capital; para ingresar con relativo éxito pero mísero reconocimiento al mercado laboral y "aumentar la productividad".

Sin embargo, es preciso apuntar tres consideraciones:

En primer lugar, no existe una investigación empírica y concreta que haya podido demostrar, más allá de la presunción, la incidencia decisiva, causal y directa de la educación en el crecimiento económico. Pero, sí existen investigaciones cuyas conclusiones muestran la manera como la integración de las tradiciones familiares, la realidad político-económica y las categorías diferenciales históricamente construidas, complejizan la mirada frente al título escolar y profundizan la diferenciación social.

Para Bourdieu, la escuela parece transmitir el saber, pero lo que hace es transmitir, también, las relaciones sociales de poder. En este sentido, los profesionales se ven obligados a entrar en la dinámica de las oportunidades sociales con sus marcas de origen, con sus apellidos de abolengo y de la universidad en que se gradúan, con sus capitales heredados (Bourdieu, 1997). La tesis doctoral que adelanta con lujo de datos el profesor Fausto Peña, comprueba este hecho para Colombia (Peña, 2012). Hay más oportunidades laborales para los egresados de la Universidad de los Andes, es cierto, pero no es solo por ser de los 
Andes: las tienen, sobre todo, por su apellido, por sus parentescos de élite, por el capital acumulado de su familia, lo cual constituye, también, una razón por la cual pueden estudiar en los Andes.

En segundo lugar, la premisa de la que se parte para asegurar la ecuación Educación = Movilidad social, puede transitar por las escalas de sofisma o anatema.

Para el PND 2006-2010, se mejora la competitividad del país si se transforma el capital humano y el capital físico. De esta manera se establece una estrecha relación de dependencia, causa y efecto, entre el crecimiento económico, la reducción de la pobreza y la superación de las desigualdades. Pero la experiencia nos dice que ello no necesariamente se aplica. Durante el gobierno del presidente Uribe, Colombia al igual que la mayoría de los países latinoamericanos, obtuvo un crecimiento económico considerable pero, al mismo tiempo, incrementó las cifras de pobres absolutos y presentó los peores índices de la región en superación de la pobreza.

Aunque Colombia tuvo cuatro años de crecimiento por encima del $5 \%$ en su PIB, durante el período 2005-2008, la bonanza no benefició a los más pobres. Por el contrario, la indigencia creció en dos puntos y el coeficiente de Gini subió una centésima. Según mostraron los indicadores, la pobreza rural se incrementó. Mientras en las ciudades la pobreza alcanzó el $40 \%$, en el campo se elevó al 63\%. Es decir, la brecha entre el campo y la ciudad se abrió aún más y el país rural se hizo más pobre y desigual.

Nuestro país se ubicó entre los más desiguales de América Latina. De acuerdo con el informe "Pobreza y precariedad urbana en América Latina y el Caribe", elaborado por la Cepal, los promedios del continente en superación de la pobreza fueron mejores que los colombianos. La pobreza disminuyó en América Latina un $10 \%$ en los últimos años, contra poco más de $7 \%$ en Colombia, en tanto la desigualdad creció. Para la Cepal, la pobreza en Colombia ronda el 50\%, solo por encima de Bolivia, Paraguay, Honduras y Nicaragua. El 70\% de la población colombiana percibe solo el 27\% del PIB, en tanto el 30\% restante recibe el 73\% (Jordán et.al, 2009).
Crecimiento económico no significa, necesariamente, reducción de pobreza e igualdad. La distribución de la riqueza obtenida con el crecimiento no siempre es "democrática".

Y, en tercer lugar, la función educativa de una escuela estrechamente ligada a los intereses de la sociedad, no debe centrar su función en la capaci-

tación para el trabajo; su función debe partir de la necesidad de formar mujeres y hombres con sentido humano; mujeres y hombres comprometidos con una ética que abarque tanto el desempeño natural o profesional como la acción personal, la acción ciudadana y, sobre todo, el cuidado frente a las decisiones que afectan a la comunidad. Si ello no es así, tendremos como resultado un mundo en donde el mercado reina y todo es viable para enriquecerse: desde la economía ilegal del narcotráfico hasta la falsa ilusión de las "pirámides".

He aquí la diferencia: se puede capacitar al "vendedor más grande del mundo" para que sea capaz de venderse a sí mismo o de reconocer ante el espejo sus propias competencias y comprarlas al mejor estilo de Og Mandino; pero este vendedor, con mucha autoestima pero sin principios, sin ética, sin sentido humano, sin seriedad profesional, puede engañar al comprador, estafarlo sin miramientos, o venderle la muerte, su propia destrucción.

\section{La "educación por competencias" en la frustrada o aplazada propuesta de reforma a la educación superior}

El "Proyecto de ley por el cual se organiza el Sistema Nacional de Educación Superior y se regula la prestación del servicio público de la Educación Superior", más conocido como "Reforma a la Educación Superior" que se presentó en el año 2011 y fue finalmente retirado del Congreso por efectos del movimiento estudiantil, tampoco escondió sus filiaciones. La expresión de la educación como derecho fue añadida a última hora, para mostrar que se cambió mucho "gracias a las reuniones con los estudiantes", pero el espíritu utilitarista y economicista, como puede 
constatarse en lo grueso del contenido de la Reforma, continuó incólume.

El término "competencias" apareció 23 veces a lo largo del texto, eliminando toda posibilidad de autonomía universitaria frente a este concepto. Se exigían para acceder a la educación superior (Art. 7); el reconocimiento de las cualidades educativas giraba en torno a ellas (Arts. 125, 133), debía ser el objetivo central de los programas técnicos, tecnólogos, profesionales y de posgrado (Arts. 60, 61, 62, 64 y 65 en donde aparecía tres veces, Art. 71 y 72); debía ser también el propósito de los doctorados y sus investigaciones (Art. 66), y se convertía en requisito del título (Art. 67), el cual debía hacerse constar en el diploma. Resultó aún más inverosímil el contenido del parágrafo en el mismo artículo 67: "Adjunto al diploma, las Instituciones de Educación Superior expedirán un documento suplemento, que contendrá información relativa al perfil y competencias desarrolladas en el programa, según reglamentación que expida el Gobierno Nacional".

¿En dónde quedaban la libertad de cátedra, el PEI, la autonomía universitaria? Las competencias, acreditadas mediante un diploma, se convertirían en la marquilla del profesional.

Continuemos: las competencias en la "Propuesta de Reforma" serían la razón de ser de los exámenes de Estado (Art. 68), el eje de todo currículo (Art. 73), la base fundamental para la evaluación de calidad y su acreditación (Art. 92) y la pieza básica para la articulación de los niveles formativos (Art. 115).

Traemos a colación una relación muy sugestiva. En la segunda mitad del siglo XX, luego de la guerra de Corea, el establecimiento militar de los Estados Unidos empezó a mirar la carrera militar como una profesión cuyo desarrollo lograba construir las tres características principales del tipo ideal: maestría, responsabilidad y vocación. Tratándose de una organización jerarquizada, la discusión formuló interesantes preguntas: ¿son profesionales los oficiales y los suboficiales? ¿Son profesionales los soldados que prestan servicio militar obligatorio y solo están de paso en el Ejército?

Las respuestas llegaron en la pluma de algunos teóricos universitarios como Samuel Huntington y Morris Janowitz (Huntington, 1964; Janowitz, 1964). Observemos sus conclusiones.

Una habilidad distintiva que compete a los militares es la administración de la violencia; pero ella corresponde a los oficiales y no a sus subalternos. Los suboficiales y los soldados son especialistas en la aplicación de la violencia pero no en su administración. Por esta razón, la profesión militar es una profesión pública burocratizada. En los suboficiales, su vocación es un oficio, pero no una profesión; diferencia reflejada en la aguda línea que se traza universalmente entre unos y otros en todas las Fuerzas Armadas del mundo. Por el contrario, la maestría del oficial le impone una especial responsabilidad social. El oficial debe ser obediente y leal con la autoridad del Estado, competente en materias militares y entrenado eficazmente para utilizar su maestría en beneficio de la seguridad del Estado. Las motivaciones del oficial son el amor técnico por su oficio y la sensación de una obligación social de utilizar este oficio para el bienestar de la sociedad. Su sentido del compromiso profesional está conformado por una ética militar que refleja un conjunto de valores y actitudes que su formación inculca con esmero. Los oficiales, por consiguiente, debían formarse en las escuelas militares, mientras los suboficiales y los soldados podrían capacitarse o entrenarse en los batallones.

Para Huntington, profesión es diferente a ocupación. La profesión significa dominio experto, compromiso, responsabilidad, autonomía y sentido de corporación; cualidades que resume en un solo término: maestría, que incluye, al mismo tiempo, la capacidad de juicio, el progreso intelectual y la adquisición de conocimientos científicos. La habilidad para el trabajo, definida como "competencias militares", no involucra precisamente una capacidad profesional, en tanto se refiere a los procedimientos y a la utilidad inmediata. En conclusión, los oficiales serán llamados a responder por su maestría, los soldados por sus competencias.

En términos de la teoría militar estadounidense, al inculcar competencias, estaríamos entrenando soldados; por el contrario, al formar en maestría, formaríamos oficiales profesionales. Lo primero puede hacerse en un batallón; lo último solo puede hacerse en escuelas. Un paralelo semejante puede ocuparse de la competencia y la maestría para las profesiones.

\section{Las estrategias del Banco Mundial y las políticas del Ministerio de Educación en Colombia}

Observemos ahora la coherencia entre el concepto citado sobre educación, las directrices del Banco Mundial y las políticas sectoriales del Ministerio de Educación Nacional de Colombia:

Un elemento de fondo que demuestra la coincidencia entre políticas nacionales y directrices internacionales, reside en el impulso brindado a la 
formación para la producción y a la preferencia de la capacitación por sobre la educación.

La perspectiva tiene dos matices. Por una parte, la educación superior se vincula con la productividad a través de la formación de un número cada vez mayor de técnicos profesionales y tecnólogos que, como aspira el Plan Sectorial vigente del Ministerio de Educación, “[...] puedan desempeñarse en los cargos para técnicos de mando medio, técnicos especializados y trabajadores calificados de la pirámide ocupacional, que hoy están vacantes u ocupados por personas poco calificadas o profesionales sobrecalificados..." (Mineducación, 2006-2010). La meta, en este sentido, es volcar la universidad hacia este propósito y dedicar mayores esfuerzos a la formación técnica y tecnológica. Es decir, según la teoría militar norteamericana, suboficiales y soldados.

Con el fin de ampliar la oferta y crecer en cobertura, el Plan de Desarrollo 2006-2010 se planteó alianzas estratégicas entre instituciones de educación superior, instituciones con programas de educación media técnica, sector productivo, gobiernos regionales y locales, SENA, y centros de innovación, productividad y desarrollo tecnológico.

En el caso del SENA, en concreto, los programas abiertos se han construido sobre la base de ciclos propedéuticos dirigidos al desarrollo de competencias y acordes con los requerimientos del sector productivo. El problema con ello es que no existe una visión de conjunto ni curricular; no existe un proyecto de formación dirigida ni un plan de estudios concreto; no impera un sistema progresivo y dinámico sobre la formación y no se obtiene la naturaleza continua de la educación formal. Dicho en otras palabras, se aplica la perspectiva de la capacitación para el empleo en reemplazo de la formación profesional con integralidad.

La reforma propuesta a la educación superior profundizaba la perspectiva. En primer lugar, equiparaba a las universidades con las instituciones de educación superior en términos generales (puros batallones), las introducía en un mismo saco y eliminaba factores diferenciales que no deben desconocerse (Capítulo II). En segundo lugar, elevaba a la categoría de "programas de grado" a los estudios técnicos profesionales y tecnológicos (Art. 60), similares a los programas profesionales. En tercer lugar, legitimaba la posi- bilidad de brindar programas por ciclos, créditos y componentes propedéuticos conducentes a títulos de educación superior (Arts. 72 y 73). En cuarto lugar, definitivamente, convertía al SENA y a las Normales en instituciones de educación superior (Art. 42) y, finalmente, establecía en cabeza de estas últimas la oferta de "Programas de Formación Complementaria" (Art. 43).

Esto último es particularmente lesivo para la profesión docente. Cualquier técnico o tecnólogo y, por supuesto, cualquier profesional, podría abordar un "Programa de Formación Complementaria" en una Normal y adquirir el título de normalista superior, o hacerlo en el SENA o en otra institución de educación superior y presentar sus créditos y cursos propedéuticos para obtener el título de "licenciado". Es, ni más ni menos, el paroxismo triunfal de los chiflados Chaparrón y Lucas. Bastaría con acercarse a una IES, pedir con algunos certificados, créditos y títulos "dígame licenciado", y le sería dicho con pergamino y acta de grado. Es, así mismo, el punto de llegada del camino abierto con el

Estatuto Docente establecido por el Decreto 1278 de 2002, que ajustó las condiciones laborales del magisterio a las políticas de flexibilización, racionalización y control, desprofesionalizó la docencia e institucionalizó la evaluación.

Volvamos al principio, al punto $\mathrm{b}$ de las estrategias sugeridas por el Banco Mundial para la educación en América Latina y el Caribe; a la construcción de mecanismos para asegurar el ajuste del sistema.

Estos programas en torno a la "racionalización o utilización óptima de recursos" se han madurado cada vez más para la educación superior. Al igual que lo sucedido en la educación básica y media, es un hecho la política de "subsidio a la demanda" por vía de los créditos. Pero, sobre todo, ha tenido lugar la ampliación drástica de cobertura sin reajuste presupuestal ni recursos adicionales. En esta dirección, el primer paso fue el asalto a la autonomía universitaria, el desconocimiento de las consultas y de los consensos en las comunidades académicas para el nombramiento de rectores leales a la política. Las históricas conquistas del movimiento estudiantil, desde el Manifiesto de Córdoba, se arrojaron por la borda y se abrió el camino a la recomposición de los Consejos Superiores. Se hizo bien la tarea y se multiplicó la 
cobertura afectando la calidad y, en ocasiones, la viabilidad misma de las universidades. Los Consejos Académicos también se portaron a la altura de lo solicitado: aceptaron la ampliación de cupos y jornadas, la apertura de nuevas carreras, la reducción en la duración de los programas académicos, el recorte de asignaturas, y facilitaron el tránsito al sistema de créditos académicos para redefinir el concepto de semestre, asegurar la culminación anticipada de la carrera y regular el comercio de la educación.

Pero, a pesar de ello, el Ministerio continuó insistiendo en la "transformación del sistema de financiamiento de la educación superior oficial", que no ha podido implementar por las continuas decisiones de la Corte Constitucional. El sueño dorado, el presupuesto "per cápita", toca azorado a la puerta. La medición a través de indicadores está lista. La relación número de alumnos y número de docentes o administrativos universitarios, como criterio para fijar tasas presupuestales que no comprenden lo complejo de la educación superior, espera en sala para desbancar a la Ley 30

de 1992. Pretende llevarse por delante las necesidades de formación e investigación que una educación de calidad exige, y olvida las notables diferencias de costos entre las universidades y su gestión histórica, las diferencias entre disciplinas, modalidades y áreas del conocimiento. La investigación, los doctorados, la calidad de los programas, el papel de la universidad en la región o incluso la acreditación, se sacrifican por la ampliación de cobertura a bajo costo e inversión.

En materia de control, la política de calidad es semejante a la impulsada para la educación básica y media: estandarización y evaluación por competencias, autoevaluación y acreditación de la calidad. Los parámetros, como sabemos, obedecen a las lógicas de superación de los mínimos y reproducen las falacias del mercado. Muy juiciosas, las universidades han intentado posicionar sus "productos educativos" entre los "clientes", dotándolos con alegados atributos de calidad y aspirando a una mayor demanda como premio, o buscando la limosna presupuestal con las nuevas reglas de juego.
El tercer aspecto no ofrece discusión ni duda. Recordémoslo. Dice el Banco Mundial: creación de observatorios del mercado laboral ofrecido por las instituciones de educación pública y privada, bajo patrones de eficiencia.

El mandato ha sido seguido en los diferentes Planes Nacionales de Desarrollo a partir del 2002, pero fue la "Revolución Educativa" quien mejor lo definió en el año 2006: "El Observatorio Laboral para la Educación es un sistema de información que reúne datos concretos para interpretar las relaciones entre el mundo de la educación superior y el mundo laboral", afirmó el Ministerio (Mineducación, 2006-2010). Se trata, como lo reconoce, de una herramienta estratégica para orientar y decidir las políticas en la educación superior. Desde luego, las artes y las humanidades corren peligro de extinción frente a las estadísticas laborales.

Finalmente, revisemos brevemente el punto d: créditos financieros educacionales orientados a subsidiar la demanda y a fortalecer la privatización de la educación.

Es claro que los créditos educativos permanecen orientados a subsidiar la demanda y a fortalecer la privatización de la educación. Los recursos contratados para educación con el propio Banco Mundial en el primer gobierno de Uribe Vélez, fueron destinados al impulso de un esquema basado en la financiación de la demanda y se convirtieron en créditos para el pago de matrículas en las instituciones de educación superior privadas propiciando, en lo estratégico, el debilitamiento de la universidad pública.

Fue ese el espíritu del Capítulo II del Proyecto de Reforma, coincidente con el sueño del Plan 2019: "Promover la participación activa del sector privado, como oferente del servicio de educación superior y como agente activo para la formulación de la política".

En estas condiciones, el Estado se convierte en una especie de banco que, en lugar de atender directamente el derecho a la educación de sus asociados, les presta para que se defiendan individualmente en el mercado de los particulares. 
He aquí, entonces, algunos puntos críticos en los cuales la influencia del Banco Mundial en las políticas educativas colombianas se hace palpable.

\section{Conclusiones principales}

Existe real coincidencia entre las políticas educativas trazadas por el Banco Mundial y las recientes políticas para la educación superior diseñadas en Colombia. El enfoque mercantilizador, articulado a las necesidades y demandas del sistema económico, se expresa en concepciones utilitaristas visibles en los Planes Nacionales de Desarrollo y fortalece las tendencias privatizadoras de la educación pública.

La educación por competencias, que vincula a la educación superior con la productividad y dedica mayores esfuerzos a la formación técnica y tecnológica, no solo concentra su mirada sobre la construcción de culturas laborales y sujetos para el exclusivo ejercicio del trabajo; es particularmente lesiva para la profesión docente.

La propuesta central con respecto a la educación superior y su ampliación de cobertura, gira en torno al otorgamiento de créditos estudiantiles. Con ello se estimula un esquema de financiación basado en la demanda, que robustece el mercado de la educación superior privada pero abandona a su suerte a las universidades públicas. El Estado se comporta como banca de "segundo piso" y renuncia a su papel garante del derecho a la educación, establecido en la Constitución Nacional.

\section{Referencias}

Atehortúa, A. (2006). La Revolución Educativa, transcurso, resultados y perspectiva. En Análisis político. Bogotá: IEPRI, Universidad Nacional de Colombia. No. 57.

Bourdieu, P. (1997). Capital cultural, escuela y espacio social. Madrid: Siglo XXI Editores.

Departamento Nacional de Planeación. (2002). Bases del Plan Nacional de Desarrollo 2002-2006. Hacia un Estado comunitario. Bogotá: Presidencia de la República - DNP.

Departamento Nacional de Planeación. (2007). Plan Nacional de Desarrollo 2006-2010. Estado comunitario: desarrollo para todos. Bogotá: Presidencia de la República - DNP.

Departamento Nacional de Planeación. (2011). Plan Nacional de Desarrollo 2010-2014. Prosperidad para todos. Bogotá: Presidencia de la República - DNP.

Estrada Álvarez, J. (2011). Organización mercantil y privatización de la educación. La mano dura de la ley. Disponible en: http://www.humanas.unal.edu.co/contextoedu/. Recuperado: 1 de noviembre de 2011
Estrada Álvarez, J. (2011). Configuraciones de política educativa neoliberal. Disponible en: http://www.humanas. unal.edu.co/contextoedu. Recuperado: 1 de noviembre de 2011.

Giddens, A. (1979). La estructura de clases en las sociedades avanzadas. Madrid: Alianza.

Holm-Nielsen, L., Blom, A. y García, P. (2003). Cómo procurar las capacidades necesarias para la economía del conocimiento. El Banco Mundial en la educación terciaria de América Latina y el Caribe. En breve, núm. 19, Banco Mundial, Washington, D.C.

Huntington, S. (1964). The soldier and the state: the theory and politics of civil military relations. New York: Vintage Books.

Inter-American Development Bank. (2000). Reforming Primary and Secondary Education in Latin America and the Caribbean. An IDB Strategy. Washington, D.C.: Sustainable Development Department Sector Strategy and Policy Papers. Disponible en: http://idbdocs.iadb.org/ wsdocs/getdocument.aspx?docnum. Recuperado: 1 de noviembre de 2011.

Janowitz, M. (1964). The military in the political development of New Nations: an essay in comparative analysis. Chicago: The University of Chicago Press.

Jordán, R. y Martínez, R. (2009). Pobreza y precariedad urbana en América Latina y el Caribe. Situación actual y financiamiento de políticas y programas. Cepal, Documentos de proyectos.

Ministerio de Educación. Revolución Educativa: Plan sectorial 2006-2010. Documento No. 8, en línea. Disponible en: http://www.mineducacion.gov.co/1621/articles/. Recuperado: 1 de noviembre de 2012.

Peña, F. (2012). Distribución social del capital escolar en Colombia [tesis doctoral]. Bogotá: Universidad Pedagógica Nacional.

Thompson, E. (1979). Tradición, revuelta y consciencia de clase. Estudios sobre la crisis de la sociedad preindustrial. Barcelona: Crítica.

Uribe Vélez, Á. (2001). Manifiesto Democrático. Nombre asignado al programa de cien puntos de la campaña política "Uribe presidente".

Uribe Vélez, Á. (2001-2002). De la incertidumbre a la confianza. Propuesta de Programa para la discusión ciudadana y bases para la discusión de una política de Estado sobre educación. Disponible en: http//www.UribePresidente. com. Recuperado: 1 noviembre de 2012.

World Bank. (1999). Educational change in Latin America and the Caribbean. World Bank, Human Development Network. Disponible en: http://www-wds.worldbank. org. Recuperado: 1 de noviembre de 2011

World Bank. (2002). Proyect appraisal document on a proprosed loan in the amount of US\$200 million to the Republic of Colombia for a higher education - improvind access project. Washington, D.C. 